\title{
OS MINERAIS PESADOS DA PRAIA GRANDE DO RODÍZIO (COLARES, SINTRA): DA FONTE AO DEPÓSITO SEDIMENTAR
}

\author{
J. CASCALHO ${ }^{(1)(2)}$, M. RIBEIRO ${ }^{(2)(3)}$, R. TABORDA $^{(2)}$, A. RODRIGUES $^{(3)}$, J. REIS $^{(1)}$, J. DUARTE $^{(3)} \&$ A. OLIVEIRA ${ }^{(3)}$
}

Resumo:

\begin{abstract}
O areal da Praia Grande apresenta frequentemente uma areia escura que se associa à elevada concentração em minerais pesados. Neste trabalho pretende-se caracterizar este fenómeno e compreender as razões associadas a esta concentração. Para cumprir este objectivo foi analisado um conjunto de amostras de sedimento recolhidas na Praia Grande e em praias da mesma célula sedimentar (São Lourenço, Foz do Lizandro, Maçãs, Pequena, Adraga, Abano e Guincho). Este conjunto de amostras foi sujeito a um tratamento laboratorial que incluiu a medição da suceptibilidade magnética, a análise granulométrica, a separação dos minerais pesados por densidade (nas dimensões compreendidas entre a areia média e fina) e a identificação das espécies transparentes ao microscópio petrográfico. Os resultados deste estudo permitem verificar que as amostras recolhidas na Praia Grande possuem uma concentração anormalmente elevada em minerais pesados, com valores entre 3 e $77 \%$, que contrasta com a concentração observada nas restantes praias onde não ultrapassa $0.5 \%$. A interpretação destes resultados sugere que a Praia Grande reúne as condições para a existência de dois tipos de processos selectivos que promovem a concentração em minerais pesados. Um, de natureza permanente, relaciona-se com o processo de transposição selectiva de partículas por deriva litoral e está dependente da presença de um promontório natural no extremo sul desta praia. O outro, esporádico no tempo, é consequência de um processo selectivo de partículas que ocorre na sequência de tempestades marítimas que induzem forte erosão do perfil da praia emersa.
\end{abstract}

Palavras-chave: areia, mineralogia, processos costeiros.

\begin{abstract}
Heavy minerals from Grande Beach (Colares, Sintra): from source to the sedimentary deposit.
The sand from Grande Beach (Sintra, Portugal) often presents a dark color due to high concentration in heavy minerals. This work pretends to characterize this phenomenon as well as to explain what causes it. To fulfill this objective, it was collected a sample set from Grande Beach and other beaches of the same sedimentary littoral cell (São Lourenço, Foz do Lizandro, Maçãs, Pequena, Adraga, Abano and Guincho). The collected samples went through several analyses techniques: measure of the magnetic susceptibility, grain size distribution, heavy mineral separation by density (between medium and fine sand grain size) and identification of the transparent heavy minerals under the petrographic microscope. The results show that the samples collected from the Grande Beach reveal a high concentration in heavy minerals, with values between 3 and $77 \%$. These values make a sharp contrast when compared with the samples from the other beaches that are always under $0.5 \%$. This suggests that the Grande Beach has ideal conditions for the development of particle sorting processes that promotes the heavy mineral concentration. One of these processes it is permanent through time and is related with the presence of a rock promontory in the southern edge of the beach. The other sorting process, is sporadic and happens during sea storms that promote the erosion of the emerged beach profile.
\end{abstract}

Keywords: sand, mineralogy, coastal processes

Received: 8 April, 2016; Accepted: 9 May, 2016

\section{INTRODUÇÃO E OBJECTIVOS}

"Thus, we live in a universe primed for complexification: hydrogen atoms form stars, stars form the elements of the periodic table, those elements form planets, which in turn form minerals abundantly. Minerals catalyze the formation of biomolecules, which on Earth led to life. In this sweeping scenario, minerals represent but one inexorable step in the evolution of a cosmos that is learning to know itself."

(R. M. HAZEN in Evolution of Minerals).

A análise da composição mineralógica dos sedimentos arenosos pode constituir uma ferramenta muito útil na interpretação dos processos da dinâmica sedimentar litoral, pelo que tem merecido a atenção de muitos investigadores. Neste contex- to, os minerais pesados são frequentemente utilizados na identificação das fontes sedimentares e dos padrões de transporte sedimentar litoral. Em determinadas situações a presença abundante destes minerais, em depósitos conhecidos por placers de minerais pesados, tem sido utilizada com o propósito específico de compreender os processos físicos que regulam a sua acumulação (KOMAR \& WANG 1984; PETERSON et al. 1986; KOMAR \& Li 1991; HAMilton \& COLLINS 1998).

Em Portugal continental as praias são essencialmente constituídas por partículas terrígenas como o quartzo, a mica e o feldspato e, também, por partículas biogénicas de natureza calcítica ou aragonítica que correspondem a fragmentos de conchas de

${ }^{1}$ Museu Nacional de História Natural e da Ciência, Universidade de Lisboa, 1250-102 Lisboa

${ }^{2}$ Instituto Dom Luiz, Faculdade de Ciências, Universidade de Lisboa, 1749-016, Lisboa

${ }^{3}$ Instituto Hidrográfico, Rua das Trinas, 49, 1249-093, Lisboa; 
diversos organismos. Perante esta composição a areia destas praias exibe cores predominantemente claras. Todavia, em determinados troços costeiros mais restritos é possível observar sedimentos arenosos ricos em minerais pesados, que são visualmente distinguidos pela sua coloração mais escura. Dentro destes casos, merece destaque, a Praia Grande localizada no concelho de Sintra onde é muito frequente observar extensas áreas cobertas de areia negra, sobretudo no seu sector sul. Em situações de pós-temporal é por vezes possível observar um depósito residual muito rico nestes minerais (Fig. 1) ao ponto de chegar a ser notícia nos meios de comunicação social, tal como é documentado na notícia publicada no jornal "Público" a 20 de Abril de 2005 (http://www.publico.pt/locallisboa/jornal/praia-grande-coberta-por-areia-negra16620).

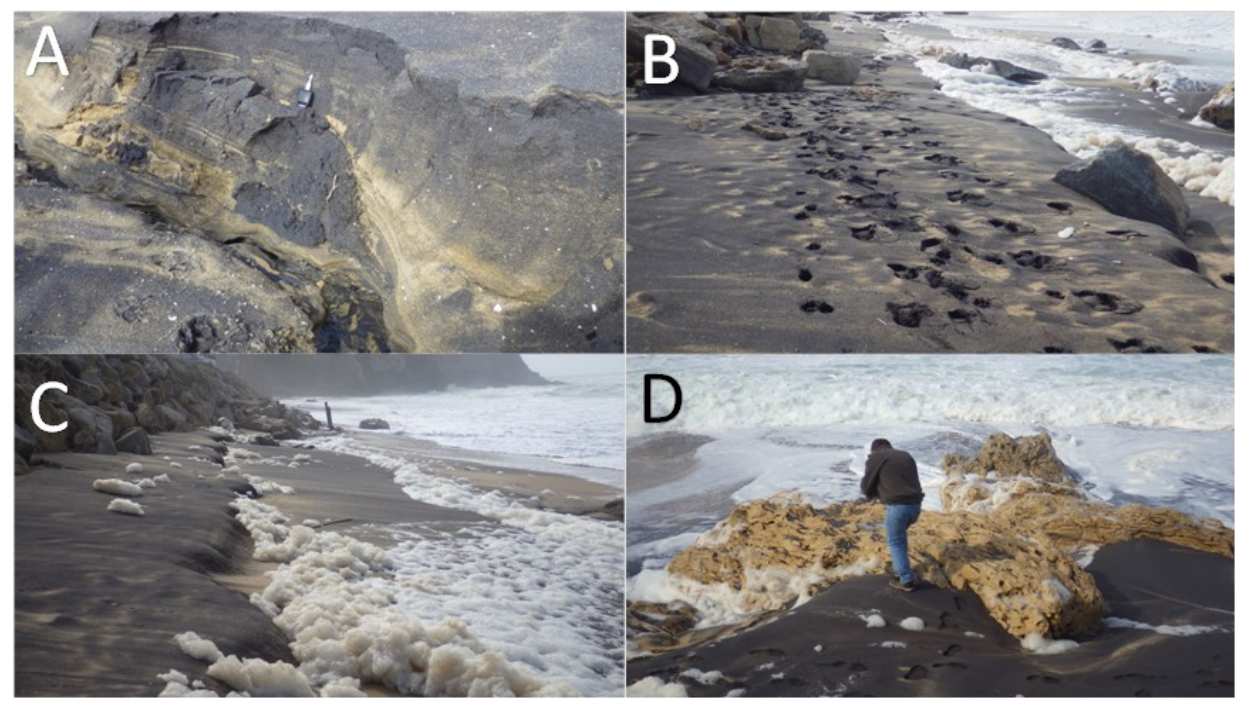

Fig. 1. Aspecto da Praia Grande a 13 de Fevereiro de 2014: A - Estrutura vertical do depósito de placer onde se observa que a espessura da camada mais rica em minerais pesados é de cerca de $30 \mathrm{~cm}$; B - Face de praia praticamente só constituída por minerais pesados; C - Escarpa de erosão formada com areia muito rica em minerais pesados; D - Aspecto da geometria superficial da areia rica em minerais pesados em redor de um afloramento de "beach rock".

Fig. 1. Visual aspect of Grande Beach at February, $13^{\text {th }}, 2014$ : A- vertical structure of the placer deposit where it is possible to observe that the upper layer richer in heavy minerals has a thickness of about $30 \mathrm{~cm} ; \mathrm{B}-$ Beach face made almost only by heavy minerals; C - Beach erosion scarp carved in heavy mineral; D - General feature of the heavy mineral placer around a beach rock outcrop.

O presente trabalho pretende caracterizar a composição mineralógica da areia da Praia Grande, explicar por que razão a concentração de minerais pesados nesta praia é geralmente muito superior à observada nas praias adjacentes e verificar se a existência desta concentração está de alguma forma relacionada com a presença das rochas do Complexo Ígneo de Sintra. Para tal foi realizada uma análise textural e composicional dos sedimentos de oito praias localizadas no troço costeiro entre São Lourenço e o Guincho (Fig. 2).

As praias referidas na Figura 2 inserem-se numa célula sedimentar litoral que se estende desde a Consolação (Peniche) ao Cabo da Roca (RIBEIRO et al. 2014). As duas praias localizadas mais a norte (São Lourenço e Lizandro) estão localizadas num troço costeiro muito recortado que tem uma orientação geral N-S e coincidem com a foz da Ribeira de Safarujo (São Lourenço) e a foz do Rio Lizandro (Foz do Lizandro). Mais para sul localizam-se as praias das Maçãs, Pequena, Grande e Adraga, num troço com orientação geral NE-SO, menos recortado relativamente ao troço anterior. A Praia das Maçãs encontra-se na foz de uma ribeira importante, a Ribeira de Colares, enquanto que a Praia da Adraga se situa na foz de um curso de água de menor importância (Ribeira da Maceira). Por último as praias do Abano e do Guincho situam-se num troço litoral com orientação aproximada NE-SO, limitado pelos cabos da Roca e Raso. Na Praia do Guincho desagua um pequeno curso de água (Ribeira da Foz do Guincho).

\section{MÉTODOS}

A amostragem de sedimento nas praias entre São Lourenço e a Adraga foi realizada no dia 11 de Fevereiro de 2015. Esta amostragem foi posteriormente complementada com sedimentos recolhidos das praias do Abano e do Guincho, a 27 de Novembro de 2015. A recolha destas amostras foi feita na face de praia durante a baixa-mar a uma cota de aproximadamente $2 \mathrm{~m}$ (relativa ao nível médio do mar). A amostragem foi realizada com um pequeno tubo de pvc previamente cortado ao meio no sentido longitudinal com cerca de $0.5 \mathrm{~m}$ de comprimento e $4.5 \mathrm{~cm}$ de diâmetro. A amostragem seguiu as recomendações que constam na nota técnica elaborada 


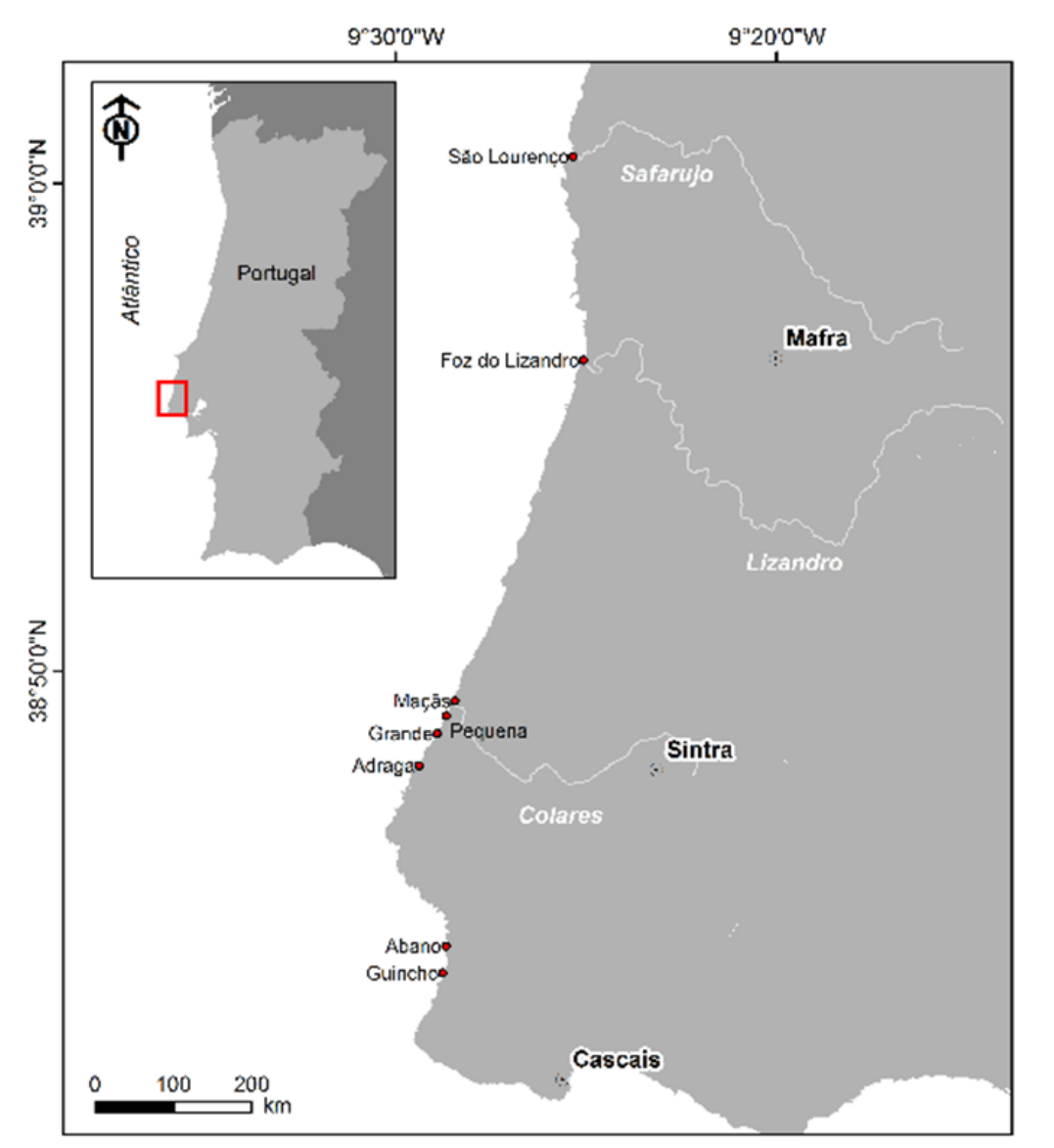

Fig. 2. Localização das praias estudadas e dos principais cursos de água.

Fig. 2. Location of the studied beaches and the main rivers and creeks.

no âmbito do projecto BEACH SAND CODE (2012), a fim de se obter uma amostra representativa das características texturais e composicionais médias de cada praia. Para além das referidas amostras, foi também analisada uma amostra colhida a 13 de Fevereiro de 2014 na Praia Grande, numa situação de pós-temporal, denominada por "Grande placer". Neste caso a amostra foi colhida manualmente e representa cerca de $30 \mathrm{~cm}$ de espessura do sedimento desta praia que na altura apresentava uma enorme concentração em minerais pesados (Fig. 1).

A granulometria das partículas com dimensão superior a $0.5 \mathrm{~mm}$ foi efetuada pelo método da peneiração, enquanto que a granulometria das partículas com dimensão inferior a este valor foi efetuada por difracção laser.

Numa primeira fase foi realizada a medição da susceptibilidade magnética dos sedimentos recolhidos cujos resultados constituem uma aproximação à concentração em minerais opacos SHANKAR et al. (1996). Antes desta operação cada amostra foi previamente lavada, seca e homogeneizada em caixas de plástico. As medições foram realizadas em 15 pontos por cada amostra utilizando um sensor de elevada resolução espacial de $3.8 \mathrm{~mm}$ (Bartington MS2E).
A identificação óptica de minerais pesados foi realizada apenas para a areia média a fina (partículas com diâmetros entre 1 e $3 \varphi$ ). Após a crivagem os sedimentos foram atacados por uma solução com $\mathrm{HCl}$ a $10 \%$ a fim de eliminar os carbonatos. Após a lavagem e secagem, efectuou-se a separação dos minerais pesados utilizando o bromofórmio. Seguidamente foi realizada uma operação de quarteamento para obter uma quantidade de "resíduo pesado" representativa da amostra e ideal para ser usada na montagem das preparações microscopias de tipo permanente, com bálsamo do Canadá $(\mathrm{n}=1.54)$. A identificação e contagem dos minerais pesados foi feita ao microscópio polarizante de luz transmitida de acordo com o método equivalente ao ribbon method (VAN HARTEN 1965 \& Galehouse 1969; 1970 citados em MANGE \& MAURER 1992), isto é, foram sistematicamente identificados todos os minerais que intersectam a parte graduada do fio E-W do retículo pela deslocação da preparação, com o auxílio de uma cremalheira ao longo de fiadas paralelas.

No caso da amostra recolhida na Praia Grande a 13 de Fevereiro de 2014 foi também realizada uma análise da composição mineralógica utilizando a difracção dos raios X. A referida amostra foi 
previamente moída e reduzida a pó $(<25 \mu \mathrm{m})$ em moinho de ágata e, posteriormente, em moinho planetário de bolas. As medições foram realizadas entre $4^{\circ}$ e $60^{\circ} 2 \theta$, com radiação $\mathrm{CuK} \alpha(45 \mathrm{Kv}$ e 40 $\mathrm{mA})$, com um passo de $0.02^{\circ}(2 \theta)$ e um tempo de contagem de $1 \mathrm{~s}$. As abundâncias relativas dos minerais identificados foram determinadas pela área do pico do difractograma e corrigidas tendo em conta os factores estimados empiricamente para os minerais identificados (MARTINS et al. 2007) em que para a ilmenite, magnetite, granada e zircão foi dada a ponderação de 1 . Aos resultados que exprimem as frequências dos 7 minerais pesados transparentes mais abundantes e, também, a percentagem de peso dos minerais pesados foi aplicada uma análise em componentes principais (ACP) com base na extracção de duas matrizes de correlação. Uma contendo todas as amostras e outra excluindo a amostra "Grande placer" (GRpla).

\section{ENQUADRAMENTO REGIONAI}

\subsection{Geologia}

A região emersa adjacente ao troço costeiro em estudo é dominada pela presença da serra de Sintra que constitui, segundo TEIXEIRA (1962), "l'élément géologique et géomorphologique le plus remarquable de la péninsule de Lisbonne". Este maciço é essencialmente constituído por rochas graníticas, sieníticas e gabro-dioríticas (MATOS Alves 1964; Wright 1969; SPARKS \& WADGE 1975; LEAL 1990) que contêm uma ampla variedade de minerais. Outro conjunto de formações com expressão significativa nas bacias hidrográficas das ribeiras que alimenta o troço litoral em estudo diz respeito ao "Complexo Basáltico de Lisboa". Neste conjunto figuram, entre outras rochas, riólitos, traquitos, traquibasaltos, basaltos e gabros, sendo os basaltos o tipo litológico mais abundante (PALÁCIOS 1985). Com excepção destes afloramentos de natureza ígnea, a área correspondente às bacias hidrográficas que drenam para o troço de litoral em estudo é dominada pela presença de formações sedimentares que vão desde o Jurássico Superior (Oxfordiano Superior) até aos depósitos sedimentares indiferenciados do Plio-Quaternário (KULLBERG \& KULLBERG 2000). Todas estas formações são, em maior ou menor grau, potenciais fontes dos minerais pesados identificados. Na Tabela 1 é apresentada a relação entre os diferentes tipos litológicos e os principais minerais pesados aí identificados de acordo com as referências consultadas.

Tabela 1. Principais rochas das bacias hidrográficas que drenam para o troço litoral em estudo e sua correspondente composição em minerais pesados.

Table 1. Heavy mineral composition of the rocks outcrops present at the watersheds that drain to the study coastal stretch.

\begin{tabular}{|c|c|c|c|}
\hline Rocha fonte & Localização & Minerais pesados principais & Referência \\
\hline Granito & Maciço de Sintra & Biotite, apatite, alanite, magnetite, zircão. & MATOS ALVES (1964) \\
\hline Sienito & Maciço de Sintra & $\begin{array}{l}\text { Anfíbola, piroxena, biotite magnetite, } \\
\text { titanite, apatite, epídoto, minério (óxido de } \\
\text { Fe-Ti?). }\end{array}$ & MATOS AlVES (1964) \\
\hline Gabro & Maciço de Sintra & $\begin{array}{l}\text { Clinopiroxena, óxidos de } \mathrm{Fe}-\mathrm{Ti} \text {, apatite, } \\
\text { anfibola castanha, biotite. }\end{array}$ & MIRANDA, (2010) \\
\hline Diorito & Maciço de Sintra & $\begin{array}{l}\text { Biotite, clinopiroxena, anfíbola castanha, } \\
\text { óxidos de Fe-Ti. }\end{array}$ & MiRANDA, (2010) \\
\hline Basalto & $\begin{array}{l}\text { Complexo vulcânico de } \\
\text { Lisboa }\end{array}$ & $\begin{array}{l}\text { Óxidos de Fe-Ti (séries magnetite- } \\
\text { ulvoespinela e ilmenite-hematite), clinopiro- } \\
\text { xenas (série diópsido-hedenbergite), anfíbo- } \\
\text { la, biotite e titanite. }\end{array}$ & PALÁCIOS (1985) \\
\hline $\begin{array}{l}\text { Rochas sedimentares } \\
\text { (Cretácico) }\end{array}$ & $\begin{array}{l}\text { Afloram em todas as } \\
\text { bacias hidrográficas que } \\
\text { drenam para o troço } \\
\text { litoral em estudo }\end{array}$ & $\begin{array}{l}\text { Frequentes: Turmalina, zircão, estaurolite, } \\
\text { granada, andaluzite, rútilo, anatase, broqui- } \\
\text { te, epídoto, distena e como escassos: titani- } \\
\text { te, apatite e anfíbola verde. }\end{array}$ & BERTHOU (1973) \\
\hline $\begin{array}{l}\text { Rochas sedimentares } \\
\text { (Cenozóico) }\end{array}$ & $\begin{array}{l}\text { Depósitos de cobertura } \\
\text { existentes sobretudo a } \\
\text { norte do maciço de Sintra }\end{array}$ & $\begin{array}{l}\text { Magnetite, andaluzite, turmalina, zircão, } \\
\text { rútilo, biotite, epídoto, granada, estaurolite }\end{array}$ & $\begin{array}{l}\text { BERTHOU (1973); DIAS } \\
\text { (1980); GALOPIM DE CAR- } \\
\text { VALHO (1994) }\end{array}$ \\
\hline $\begin{array}{l}\text { Contacto ígneas-sedimentares } \\
\text { (maciço de Sintra - rochas } \\
\text { sedimentares do Jurássico) }\end{array}$ & $\begin{array}{l}\text { Periferias meridional e } \\
\text { oriental do maciço de } \\
\text { Sintra }\end{array}$ & $\begin{array}{l}\text { Pirite, magnetite, granada (série grossulária- } \\
\text { andradite), anfíbola (pargasite) e clinopiro- } \\
\text { xena (diópsido) }\end{array}$ & $\begin{array}{l}\text { REAL (1951); MATOS } \\
\text { ALVES (1964) }\end{array}$ \\
\hline $\begin{array}{l}\text { Contacto ígneas-sedimentares } \\
(\mathrm{CVL} \text { - rochas sedimentares } \\
\text { do Cretácico) }\end{array}$ & $\begin{array}{l}\text { Monte Suímo e Lomba } \\
\text { dos Pianos }\end{array}$ & Granada (almandina e grossulária) & PALÁCIOS (1985) \\
\hline
\end{tabular}

\subsection{Forçamento oceanográfico}

A costa oeste portuguesa está sujeita a um regime de maré mesotidal do tipo semi-diurno. De acordo com COSTA \& ESTEVES (2009), o clima de agitação ao largo é caracterizado por uma altura significativa média de $2 \mathrm{~m}$ com um desvio padrão de $1.1 \mathrm{~m}$ (valores médios anuais). Os valores extremos referentes a esta altura variam entre 0.34 e 13 m. No que se refere aos rumos verifica-se que os mais frequentes provêm do octante noroeste $(69$ $\%$ ), seguindo-se os rumos de oeste (cerca de $20 \%$ ) e de norte (cerca de $9 \%$ ). O padrão de distribuição da altura significativa (Hs) e do período de pico (Tp) têm uma distribuição regulada pela sucessão das estações do ano. Assim, durante o Verão marítimo (entre Abril e Setembro), os valores medianos da Hs e do Tp são inferiores a $2 \mathrm{~m}$ e a $11 \mathrm{~s}$, respectivamente. Os valores mais elevados ocorrem no 
Inverno marítimo, entre Dezembro e Março, onde a mediana da Hs se situa entre os 2 e os 3 m enquanto que o valor mediano do Tp se situa entre 12 e $13 \mathrm{~s}$ (TABORDA et al. 2013). Cabe aqui referir o facto do Inverno de 2013/2014 ter sido caracterizado pela ocorrência de ventos fortes e ondas muito altas geradas por vários ciclones extratropicais que se desenvolveram no Atlântico Norte. Entre os vários ciclones que afectaram o litoral oeste de Portugal continental durante este Inverno destaca-se a ocorrência do ciclone denominado Christina que atingiu a sua máxima expressão no início de Janeiro de 2014 e, também, o ciclone Stephanie que ocorreu no início de Fevereiro de 2014 (Diogo et al. 2014).

\section{RESULTADOS}

\subsection{Textura}

Os parâmetros estatísticos exibidos na Tabela 2 (média e desvio padrão; FOLK \& WARD 1957) mostram que, em geral, as dimensões médias das partículas caiem no domínio da areia grosseira (entre 0 e 1 $\varphi$ ). São excepção, o sedimento de São Lourenço que corresponde a areia muito grosseira e o sedimento da Praia Grande placer classificado como areia média. Quanto ao desvio padrão verifica-se que este varia entre o muito bem calibrado (Praia Pequena) e o moderadamente bem calibrado (casos das praias de São Lourenço, Foz do Lizandro e Maçãs).

Tabela 2. Média, desvio padrão e classificação dos sedimentos das praias (FOLK \& WARD 1957). Valores em unidades $\varphi$.

Table 2. Beach sediment grain size medium, sorting and correspondent classification according to FOLK \& WARD (1957). Values in $\varphi$ units.

\begin{tabular}{|c|c|c|c|c|}
\hline Praia & \multicolumn{2}{|c|}{ Média e classificação } & \multicolumn{2}{c|}{ Desvio padrão e classificação } \\
\hline São Lourenço (SL) & -0.58 & Areia muito grosseira & 0.70 & modera. bem calibrado \\
\hline Foz do Lizandro (FL) & 0.53 & Areia grosseira & 0.60 & modera. bem calibrado \\
\hline Maçãs (MA) & 0.48 & Areia grosseira & 0.66 & modera. bem calibrado \\
\hline Pequena (PEQ) & 0.80 & Areia grosseira & 0.34 & muito bem calibrado \\
\hline Grande (GR) & 0.92 & Areia grosseira & 0.37 & bem calibrado \\
\hline Grande placer (GRpla) & 1.53 & Areia média & 0.49 & bem calibrado \\
\hline Adraga (ADA) & 0.83 & Areia grosseira & 0.37 & bem calibrado \\
\hline Abano (ABA) & 0.84 & Areia grosseira & 0.42 & bem calibrado \\
\hline Guincho (GUI) & 0.76 & Areia grosseira & & bem calibrado \\
\hline
\end{tabular}

\subsection{Susceptibilidade magnética}

Na Figura 3 é possível verificar que os valores desta grandeza física são extremamente elevados no caso da amostra GRpla. Esta amostra apresenta valores em cerca de dez vezes maiores quando comparados com os da amostra GR que, por sua vez, se destaca das restantes que revelam valores residuais de susceptipilidade magnética.

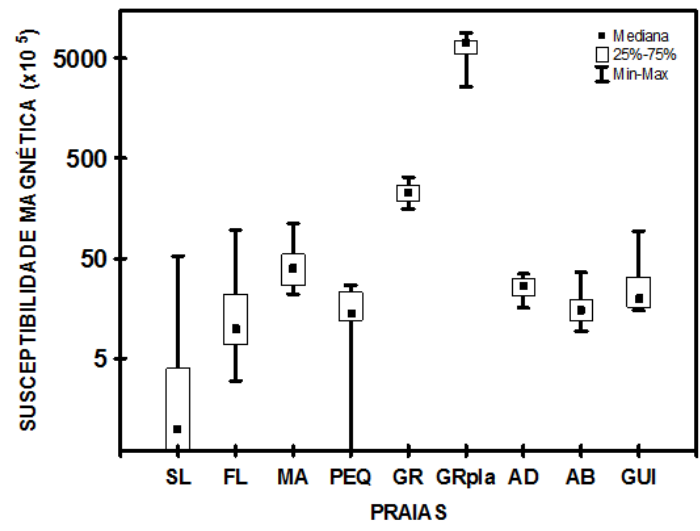

Fig. 3. Valores da mediana, quartis 25 e 75 e máximomínimo referentes à susceptibilidade magnética (valores correspondentes a 15 medições por amostra). Os valores estão representados em escala logarítmica (SL - São Lourenço, FL - Foz do Lizandro, MA - Maçãs, PEQ - Pequena, GR - Grande, GRpla - Grande placer, ADA - Adraga, ABA - Abano, GUI - Guincho).

Fig. 3. Median, percentiles $25^{\text {th }}$ and $75^{\text {th }}$ and maximumminimum of the magnetic susceptibility (values correspondent to 15 measures per sample). The values are represented on a logarithmic scale (SL - São Lourenço, FL - Foz do Lizandro, MA - Maçãs, PEQ - Pequena, GR - Grande, GRpla - Grande placer, ADA - Adraga, ABA - Abano, GUI - Guincho).

\subsection{Minerais pesados}

Os valores da percentagem de peso dos minerais pesados ponderada no sedimento total são apresentados na Tabela 3. Os resultados mostram que a maior parte das praias possui concentrações relativamente baixas nestes minerais (inferiores a $0.5 \%$ ), com excepção das amostras recolhidas na Praia Grande que contêm cerca de $3 \%$ (amostra GR) e cerca de $77 \%$ (amostra GRpla).

Tabela 3. Valores percentuais da percentagem de peso minerais pesados (MP \%) ponderados no peso total do sedimento.

Table 3. Heavy mineral weight percentages (MP\%) weighted on the total sediment weight.

\begin{tabular}{|c|c|}
\hline Praia & MP \% \\
\hline São Lourenço (SL) & 0.03 \\
\hline Foz do Lizandro (FL) & 0.10 \\
\hline Maçãs (MA) & 0.18 \\
\hline Pequena (PEQ) & 0.31 \\
\hline Grande (GR) & 3.14 \\
\hline Grande placer (GRpla) & 76.64 \\
\hline Adraga (ADA) & 0.42 \\
\hline Abano (ABA) & 0.17 \\
\hline Guincho (GUI) & 0.10 \\
\hline
\end{tabular}

A identificação óptica dos minerais transparentes permitiu reconhecer a existência de 14 espécies: turmalina (TUR), clinopiroxena (CPX), granada (GRA), estaurolite (EST), andaluzite (AND), anfíbola (ANF), zircão (ZIR), epídoto (EPI), rútilo (RU), titanite (TIT), biotite (BIO), apatite (APA), distena (DIS) e silimanite (SIL). Na Figura 4 são 
apresentadas apenas as espécies com percentagens mais significativas (com frequências medianas iguais ou superiores a $0.3 \%$ ).

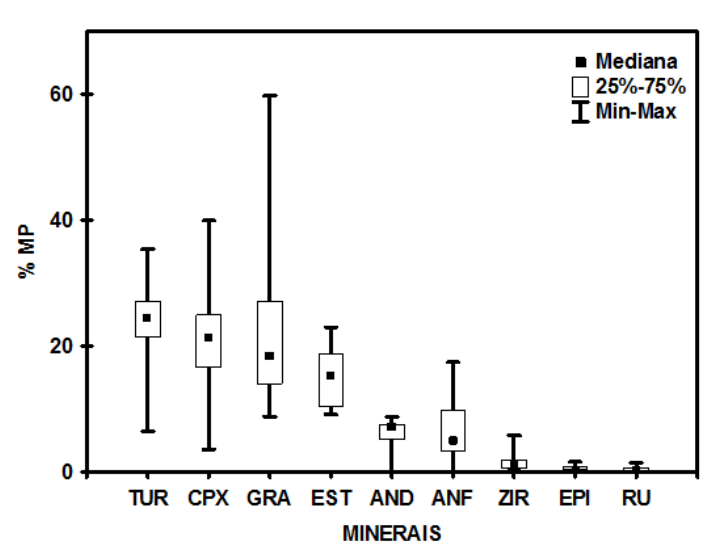

Fig. 4. Percentagem principais espécies de minerais pesados transparentes, ordenadas segundo valores decrescentes da mediana (TUR - turmalina, CPX - clinopiroxena, GRA granada, EST - estaurolite, AND - andaluzite, ANF - anfíbola, ZIR - zircão, EPI - epídoto, RU - rútilo).

Fig. 4. Main heavy mineral transparent species ordered by their median decreasing frequency (TUR - tourmaline, CPX - clinopyroxene, GRA - garnet, EST - staurolite, AND - andalusite, ANF - amphibole, ZIR - zircon, EPI - epidote, RU - rutile).
A figura 5 mostra o padrão de distribuição das 7 espécies transparentes mais comuns (da TUR até ao ZIR, inclusive) ao longo do troço costeiro analisado. É possível verificar, com a excepção da amostra GRpla, que existe uma distribuição muito homogénea destes minerais. Verifica-se assim que o cortejo dominante é invariavelmente composto pela TUR, CPX, GRA e EST. Esta homogeneidade é apenas quebrada pelo caso da amostra GRpla onde a frequência conjunta de GRA, EST e de ZIR representa cerca de $90 \%$ do cortejo transparente dominante e, também, pela presença abundante de CPX na amostra FL.

As espécies minerais identificadas apresentam ao microscópio petrográfico um leque muito variado de características observáveis (nomeadamente a sua cor em luz simplesmente polarizada e o seu grau de desgaste) que são consequência de um conjunto de processos geológicos que operam desde a origem (fonte) até ao local de sedimentação (que no caso deste trabalho é a praia). Dada a ampla variabilidade destas características optou-se por representar na Tabela 4 apenas os aspectos visuais mais comuns das espécies mineralógicas referidas na Figura 5.

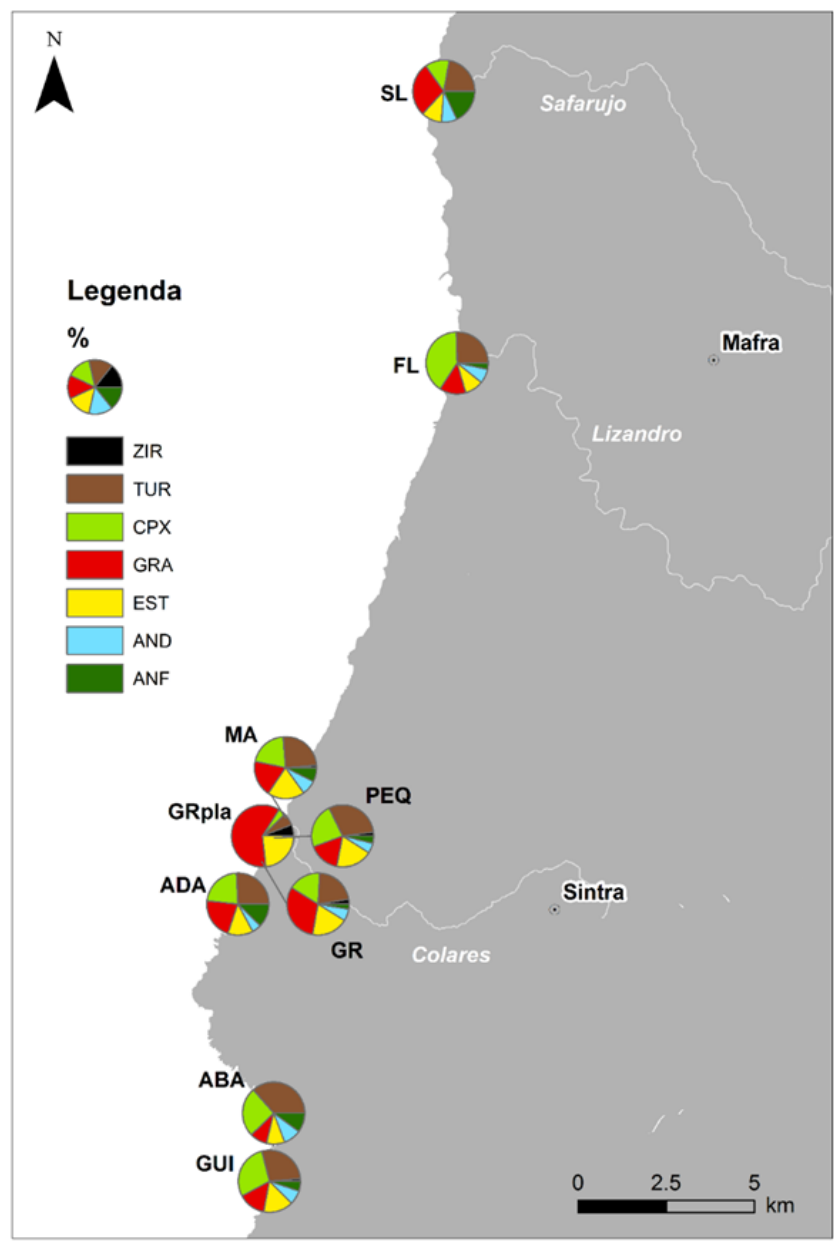

Fig. 5. Padrão de distribuição dos principais minerais pesados transparentes nas 8 praias estudadas. Minerais pesados: ZIR - zircão, TUR - turmalina, CPX clinopiroxena, GRA - granada, EST - estaurolite, AND - andaluzite, ANF - anfíbola. Praias: SL - São Lourenço, FL - Foz do Lizandro, MA - Maças, PEQ - Pequena, GR - Grande, GRpla - Grande placer, ADA - Adraga, ABA - Abano, GUI- Guincho.

Fig. 5. Distribution pattern of the main transparent heavy minerals on the 8 studied beaches. Heavy minerals: ZIR - zircon, TUR - tourmaline, CPX - clinopyroxene, GRA - garnet, EST - staurolite, AND - andalusite, ANF amphibole. Beaches: SL - São Lourenço, FL - Foz do Lizandro, MA - Maças, PEQ - Pequena, GR - Grande, GRpla - Grande placer, ADA - Adraga, ABA - Abano, GUI- Guincho. 
Tabela 4. Aspectos visuais mais comuns das espécies de minerais pesados transparentes principais. As imagens foram obtidas ao microscópio petrográfico em luz simplesmente polarizada (barra $=0.5 \mathrm{~mm}$ ). Table 4. Visual aspect of the main transparent heavy minerals. The images where captures under the petrographic microscope using plane polarized light $(\mathrm{bar}=0.5 \mathrm{~mm})$.

Clinopiroxena

Excepcionalmente foi feita uma análise da composição mineralógica da amostra GRpla através da difracção de raios $\mathrm{X}$, uma vez que esta amostra apresenta uma concentração em minerais pesados muito elevada (cerca de $77 \%$ ). Os resultados mostram que os sedimentos são essencialmente constituídos por ilmenite ( $36 \%)$ e magnetite $(29 \%)$ e, em quantidades menores, por hematite $(11 \%)$, granada $(8 \%)$ e zircão $(6 \%)$.

\subsection{Análise em componentes principais}

A primeira extracção dos componentes principais com base em 8 amostras (com a exclusão da amostra GRpla) permite verificar que os 2 primeiros componentes (de um total de 7) explicam $75 \%$ da variância total. O primeiro componente explica 49 $\%$ da variância e tem um valor próprio de 3.9. Já o segundo componente explica $26 \%$ da variância e tem um valor próprio de 2.1. A projecção das amostras num gráfico de dispersão (definido pelos dois primeiros componentes) mostra que estas são muito semelhantes sendo apenas de notar a excentricidade das amostras GR e SL relativamente ao grupo (maioritário) formado pelas restantes amostras (Fig. 6A). Na Figura 6B é possível verificar que os minerais de maior densidade (ZIR, GRA e EST) e a percentagem em minerais pesados (\%MP) se projectam todos no lado esquerdo do diagrama (no terceiro e quarto quadrantes) opondo-se aos restantes minerais que se projectam nos quadrantes opostos. 


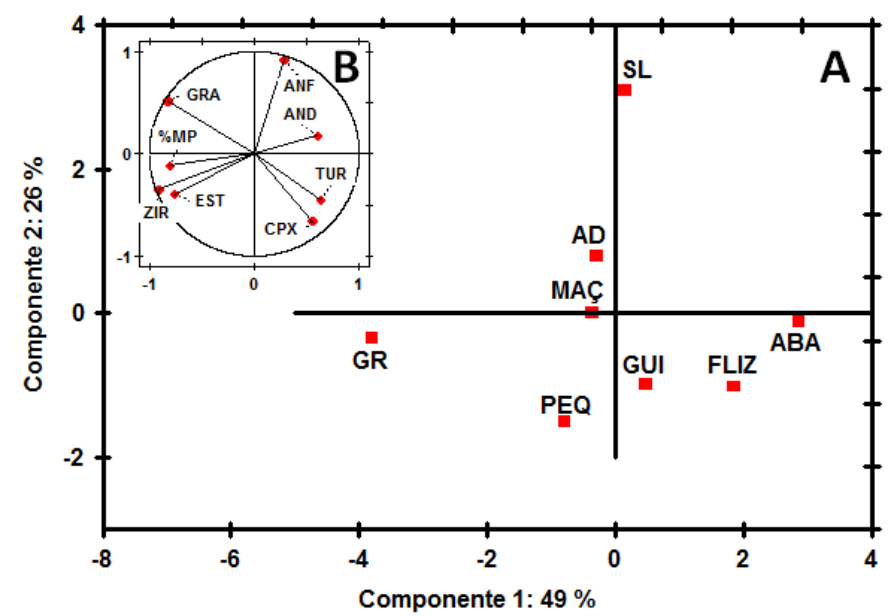

Fig. 6. A: Diagrama mostrando a projecção das amostras no espaço definido pelos dois primeiros componentes. No eixo das abcissas o primeiro componente tem $49 \%$ de variância associada e no eixo das ordenadas o segundo componente tem $26 \%$ de variância associada. B: Ponderações dos minerais pesados transparentes mais importantes (incluindo a percentagem de minerais pesados - \% MP) segundo os mesmos componentes.

Fig. 6. A: Two-dimensional scatter plot defined by the two principal components showing the samples displacement. On the x-axis the first component has $49 \%$ of the explained variance and on the y-axis the second component has $26 \%$ of the explained variance. B: Main transparent heavy mineral loadings (included the weight heavy mineral percentage - \%MP) according to the same components.

A segunda extracção dos componentes principais (que inclui todas as amostras anteriores mais a denominada por GRpla) permite verificar que os 2 primeiros componentes explicam 89 \% da variância total. O primeiro componente explica $74 \%$ da variância e tem um valor próprio de 5.9. O segundo componente explica $15 \%$ da variância e tem um valor próprio de 1.2. Os resultados mostram que a grande maioria das amostras é muito semelhante entre si sendo apenas de notar a excentricidade das amostras GRpla e SL relativamente ao grupo (maioritário) formado pelas restantes amostras (Fig. 7A). Na Figura 7B é possível verificar que os minerais de maior densidade (ZIR, GRA e EST) e a percentagem em minerais pesados (\%MP) se projectam todos no lado esquerdo do diagrama (no terceiro e quarto quadrantes e em posições muito próximas) opondo-se aos restantes minerais que se projectam nos quadrantes opostos.

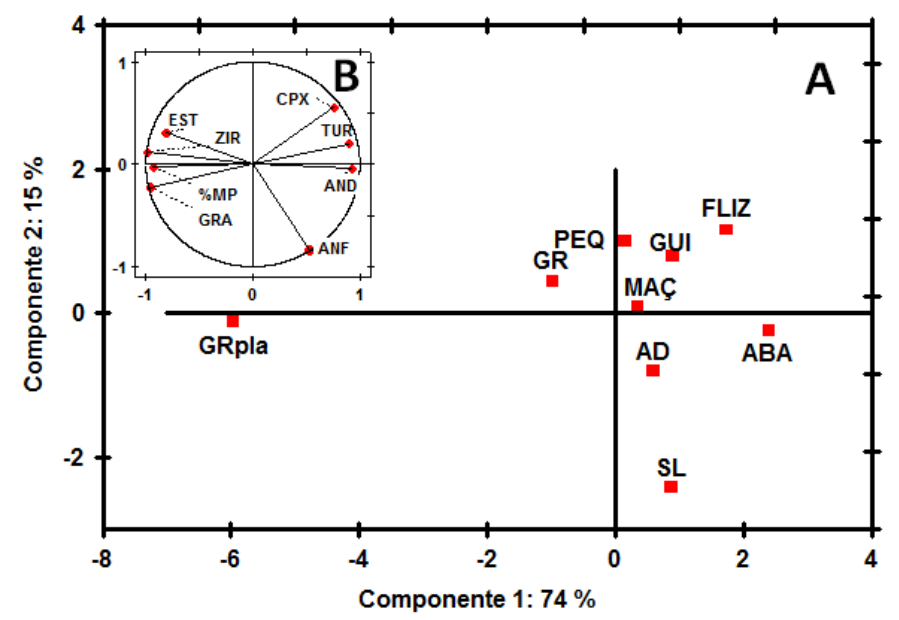

Fig. 7. A: Projecção das amostras no espaço definido pelos dois primeiros componentes. No eixo das abcissas o primeiro componente tem 74 \% de variância associada e no eixo das ordenadas o segundo componente tem $15 \%$ de variância associada. B: Ponderações dos minerais pesados transparentes mais importantes (incluindo a percentagem de minerais pesados - \% MP) segundo os mesmos componentes.

Fig. 7. A: Two-dimensional scatter plot defined by the two principal components showing the samples displacement. On the $\mathrm{x}$-axis the first component has $74 \%$ of the explained variance and on the $\mathrm{y}$-axis the second component has $15 \%$ of the explained variance. B: Main transparent heavy mineral loadings (included the weight heavy mineral percentage - \%MP) according to the same components. 


\section{DISCUSSÃO}

A composição mineralógica de um depósito sedimentar como o da Praia Grande é consequência dos processos de fornecimento e distribuição sedimentares. Os resultados da composição em minerais pesados das praias estudadas mostram que apesar da natureza geológica das fontes ser muito diversificada o cortejo mineralógico é muito semelhante (Tab. 1 e Fig. 5). De facto, os minerais mais abundantes estão presentes em rochas de natureza litológica muito diversa, pelo que os minerais identificados não podem ser usados como indicadores de proveniência. Conclui-se assim que os resultados não sustentam a hipótese de que a assinatura mineralógica específica da Praia Grande possa ser o resultado de uma fonte sedimentar relacionada com as rochas do Complexo Ígneo de Sintra e das rochas do correspondente encaixante sedimentar metamorfizado.

Assim sendo, a composição mineralógica do sedimento da Praia Grande estará, então, relacionada com um conjunto de processos físicos que favorecem a concentração dos minerais pesados. Este troço costeiro enquadra-se numa célula sedimentar que se estende desde da Praia da Consolação (concelho de Peniche) até à Praia do Guincho (concelho de Cascais) onde a deriva litoral apresenta uma resultante dirigida para sul (RIBEIRO, 2014). No troço costeiro referente ao presente trabalho as praias ocupam reentrâncias da linha de costa muitas vezes na dependência directa de desembocaduras de linhas de água. $\mathrm{Na}$ maior parte dos casos a maior capacidade de retenção de sedimentos associa-se às características do obstáculo sotamar. Na Praia Grande, este limite, está associado ao promontório do Calhau do Corvo que apresenta características peculiares, uma vez que é formado por rochas compactas carbonatadas do Cretácico inferior que exibem uma estratificação quase vertical com direcção perpendicular à orientação geral da linha de costa. Esta particularidade geomorfológica coloca a face desta praia muito afastada da zona onde é feita a transposição sedimentar na direcção paralela à linha de costa. Desta forma, a Praia Grande funciona como um "filtro" dos sedimentos que são transportados por deriva litoral, promovendo a concentração dos minerais mais densos em detrimento dos mais leves.

A este processo de escala temporal mais alargada sobrepõe-se o efeito das tempestades, que actua a uma escala temporal mais pequena, e que é responsável pela geração do depósito muito rico em minerais pesados (placer de minerais pesados). A amostra que representa esta situação (GRpla) contém um cortejo de minerais pesados essencialmente formado pela ilmenite, magnetite, hematite, granada, estaurolite e zircão, cujos valores de densidade são os mais elevados entre os minerais identificados nas praias estudadas. A formação dos placers de minerais pesados (na sequência de episódios de tempestade) encontra-se descrita em numerosos trabalhos (RAO 1957; KOMAR \& WANG 1984; CASCALHO \& TABORDA 2006; KOMAR 2007) cuja ocorrência tem lugar devido a condições excepcionais de selecção de partículas de acordo com as suas dimensões e densidades (KUDRASS 1987).

\section{CONCLUSÕES}

Para a maioria das praias estudadas a composição mineralógica dos sedimentos não apresenta diferenças significativas. Todavia, existem três excepções relacionadas com as amostras recolhidas nas praias de São Lourenço e Grande. No caso da amostra recolhida na Praia de São Lourenço as diferenças são de carácter textural devido à presença de sedimentos com uma média granulométrica de areia muito grosseira. No caso das duas amostras recolhidas da Praia Grande estas diferenças reportam-se à presença de uma elevada quantidade de minerais pesados que varia entre cerca de $3 \%$, numa situação normal, e cerca 77 \% numa situação de pós-temporal. Estes valores que exprimem a concentração em minerais pesados são interpretados como consequência de processos selectivos de partículas arenosas que actuam com muita eficiência nesta praia, devido ao seu particular enquadramento geomorfológico. Muito provavelmente, o valor de $3 \%$, reflecte o funcionamento da Praia Grande como um "filtro" de partículas arenosas que são transportadas por deriva litoral, promovendo a concentração dos minerais mais densos em detrimento dos mais leves, processo que se desenvolve de uma forma contínua ao longo do tempo. Por sua vez, o valor de $77 \%$, será consequência de uma situação extrema na sequência da ocorrência de tempestades muito energéticas. Nestas alturas (esporádicas no tempo) o process o selectivo é muito eficiente originando o desenvolvimento na praia de um depósito residual muito rico em minerais pesados (placer de minerais pesados) que é essencialmente caracterizado pela presença de ilmenite, magnetite, hematite, granada, estaurolite e zircão.

\section{BIBLIOGRAFIA}

BEACH SAND CODE 2012. Beach sediment sampling and processing guidelines. Relatório técnico do projecto "Beach Sand Code", 9p., disponível em http:// sandcode.fc.ul.pt/References/sampling\% 20guidelines.pdf

Berthou, P. Y. 1973. Le Cénomanien de l'Estremadure portugaise. Memórias dos Serviços Geológicos de Portugal, N. S. 23, 169 p

CASCALHO, J. \& TABORDA, R. 2006. Heavy mineral placer formation: an example from Algarve, Portugal. Journal of Coastal Research, SI 39, 246-249.

CostA, M. \& Esteves, R. 2009. Clima de agitação marítima na costa oeste de Portugal Continental. Comuni- 
cações das XI Jornadas Técnicas de Engenharia Naval, Instituto Superior Técnico, Lisboa, 15 p.

DiAS, M. H. 1980. A plataforma Litoral a norte de Sintra. Estudo dos depósitos de cobertura. Relatório $n{ }^{\circ} 11$ da Linha de Acção de Geografia Física do INIC. Centro Estudos Geográficos da Universidade de Lisboa, 59 p.

Diogo Z., Bastos, A., Lira, C., Taborda, R., Freire de Andrade, C., Silveira, T. M., Ribeiro, M., Silva A. N., CarapuÇo, M. M., Pinto, C. A. \& Freitas, M. C. 2014. Morphological impacts of Christina storm on the beaches of the central western Portuguese coast. Comunicações Geológicas, 101, Especial III, 1445-1448.

FOLK R. L. \& WARD W. C. 1957. Brazos River bar: a study in the significance of grain size parameters. Journal of Sedimentary Petrology, 27,3-26.

Galopim de Carvalho, A. M. 1994. O Cenozóico continental a norte da serra de Sintra (estudo tectonosedimentar). Memórias de Geociências do Museu Nacional de História Natural da Universidade de Lisboa, 1, 89 p.

Hamilton, N. T. M. \& Collins, L., B. 1998. Placer Formation in a Holocene Barrier System, Southwestern Australia. Journal of Coastal Research, 14, 240255.

HAZEN, R. M. 2010. Evolution of Minerals. Scientific American, 302, 3, 58-65.

KOMAR, P.D., 2007. The entrainment, transport and sorting of heavy minerals by waves and currents. In: M.A. Mange \& D.T. Wright (Eds.), Heavy minerals in use, Developments in Sedimentology 58, 3-48, Amsterdam, Elsevier.

Komar, P. D. \& WANG, C. 1984. Processes of Selective Grain Transport and the Formation of Placers on Beaches. The Journal of Geology, 92.6, 637-655.

Komar, P. D. \& LI, M. Z. 1991. Beach Placers at the Mouth of the Columbia River, Oregon and Washington. Marine Mining, 10, 171-187.

KUDRASS, H. R. 1987. Sedimentary models to estimate the heavy-mineral potential of shelf sediments. In: P. G. Teleki et al. (eds.), Marine Minerals, 39-56, Dordrecht, D. Reidel Publishing Company.

Kullberg, M. C. \& KullberG, J. C. 2000. Tectónica da região de Sintra. In: Tectónica das regiões de Sintra e Arrábida. Memórias de Geociências do Museu Nacional de História Natural da Universidade de Lisboa, 2, 1-34.

LEAL, N. F. G. 1990. O maciço eruptivo de Sintra. Novos dados de natureza petrográfica e geoquímica. Dissertação no âmbito das Provas de Aptidão Pedagógica e Científica. Departamento de Geologia da Faculdade de Ciências da Universidade de Lisboa, 49 p. e vários anexos.

MAnge, M.A. \& MAurer, H. F. W. 1992. Heavy minerals in Colour. London, Chapman and Hall, 147 p.
Matos Alves, C. A., 1964. Estudo petrológico do maciço eruptivo de Sintra. Revista da Faculdade de Ciências da Universidade de Lisboa, 2. ${ }^{\mathrm{a}}$ Série, C, XII (2), 124-289.

Martins, V., Dubert, J., Jouanneau, J.M., Weber, O., Silva, E.F., Patinha, C., Dias, J.M.A. \& RochA, F. 2007. A multiproxy approach of the Holocene evolution of shelf-slope circulation on the NW Iberian Continental Shelf. Marine Geology, 239, 1-18.

Miranda, R. M. L. 2010. Petrogenesis and Geochronology of the Late Cretaceous Alkaline Magmatism in the West Iberian Margin. Dissertação de doutoramento. Faculdade de Ciências da Universidade de Lisboa, 367 p. e vários anexos.

PalÁcios, T. 1985. Petrologia do Complexo Vulcânico de Lisboa. Dissertação de doutoramento. Faculdade de Ciências da Universidade de Lisboa, 290 p.

Peterson, C. D., Komar, P. D. \& Scheidegger, K. 1986 Distribution, geometry, and origin of heavy mineral placer deposits on Oregon beaches. Journal of Sedimentary Petrology, 56, 67-77.

RAO, C. B. 1957. Beach erosion and concentration of heavy minerals sands. Journal of Sedimentary Petrology, 27, 143-147.

REAL, F. 1951. Algumas observações sobre a auréola metamórfica da Serra de Sintra. Boletim do Museu e Laboratório Mineralógico de Geológico da Faculdade de Ciências da Universidade de Lisboa, 19, 99-106.

Ribeiro, M., TABorda, R., Rodrigues, A. \& Silveira, T. 2014. Insights on sediment bypassing at headlandbay beaches: an example at the Portuguese west coast. Actas das 3. ${ }^{\text {as }}$ Jornadas de Engenharia Hidrográfica. Instituto Hidrográfico, Lisboa, 301-304.

Shankar, R., ThOMPson, R. \& Prakash, T.N. 1996. Estimation of heavy and opaque mineral contents of beach and offshore placers using rock magnetic techniques. Geo-Marine Letters, 16:(4), 313-318.

SpARKS, R. S. J. \& WAdGE, G. 1975. Geological and geochemical studies of the Sintra alkaline igneous complex, Portugal”. Bulletin Volcanologique, 39 (3), 383-406

Taborda, R., Andrade, C., Silva, A. M., Silveira, T.M. \& LIRA, C. 2013. Entregável 1.1.7.a: Caracterização do clima de agitação ao largo. Relatório Técnico, Projeto Criação e implementação de um sistema de monitorização no litoral abrangido pela área de jurisdição da Administração da Região Hidrográfica do Tejo. FFCUL/APA, I.P., Lisboa, 22 p. + anexos (não publicado).

TEIXEIRA, C. 1962. La structure annulaire de Sintra, Sines et Monchique. Estudo cientifico oferecido em homenagem ao prof. Carrington da Costa. Junta de Investigação do Ultramar, Lisboa, 461-493.

WRIGHT, J. B. 1969. Re-interpretation of a mixed petrographic province - The Sintra Intrusive Complex (Portugal) and related rocks. Geologische Rundschau, 58 (2), Estugarda, 338-559. 